\title{
Article \\ Clinical Implications of Neurological Comorbidities and Complications in ICU Patients with COVID-19
}

\author{
Jaeseok Park ${ }^{1}\left(\mathbb{D}\right.$, , Yong-Shik Kwon $^{1}$, Hyun-Ah Kim ${ }^{2}{ }^{\circledR}$, Doo-Hyuk Kwon ${ }^{3,4}$, Jihye Hwang ${ }^{3}{ }^{\mathbb{D}}$, Seong-Hwa Jang ${ }^{5}$, \\ Hyungjong Park ${ }^{5}$, Sung-Il Sohn ${ }^{5}{ }^{-}$, Huimahn Alex Choi ${ }^{6}$ and Jeong-Ho Hong ${ }^{5, *(1)}$
}

1 Department of Internal Medicine, Division of Pulmonology, Keimyung University Dongsan Hospital, Keimyung University School of Medicine, Daegu 42601, Korea; mdpjs79@naver.com (J.P.); kwonys0515@naver.com (Y.-S.K.)

2 Division of Infectious Disease, Department of Internal Medicine, Keimyung University Dongsan Hospital, Keimyung University School of Medicine, Daegu 42601, Korea; hyunah1118@dsmc.or.kr

3 Department of Neurology, Daegu Dongsan Hospital, Keimyung University School of Medicine, Daegu 41931, Korea; kspy1d@naver.com (D.-H.K.); jhhwang0110@gmail.com (J.H.)

4 Department of Neurology, Yeungnam University Hospital, Yeungnam University School of Medicine, Daegu 42415, Korea

5 Department of Neurology, Keimyung University Dongsan Hospital, Keimyung University School of Medicine, Daegu 42601, Korea; seonghwajang0416@gmail.com (S.-H.J.); hjpark209042@gmail.com (H.P.); sungil.sohn@gmail.com (S.-I.S.)

6 Department of Neurology and Neurosurgery, McGovern Medical School, University of Texas Health Science Center, Houston, TX 77030, USA; Huimahn.A.Choi@uth.tmc.edu

* Correspondence: neurohong79@dsmc.or.kr

check for updates

Citation: Park, J.; Kwon, Y.-S.; Kim, H.-A.; Kwon, D.-H.; Hwang, J.; Jang, S.-H.; Park, H.; Sohn, S.-I.; Choi, H.A.; Hong, J.-H. Clinical Implications of Neurological Comorbidities and Complications in ICU Patients with COVID-19. J. Clin. Med. 2021, 10, 2281. https://doi.org/10.3390/ jcm10112281

Academic Editor: Vito Racanelli

Received: 8 April 2021

Accepted: 21 May 2021

Published: 25 May 2021

Publisher's Note: MDPI stays neutral with regard to jurisdictional claims in published maps and institutional affiliations.

Copyright: (c) 2021 by the authors. Licensee MDPI, Basel, Switzerland. This article is an open access article distributed under the terms and conditions of the Creative Commons Attribution (CC BY) license (https:/ / creativecommons.org/licenses/by/ $4.0 /)$.

\begin{abstract}
Clinical implications of neurological problems during intensive care unit (ICU) care for coronavirus disease 2019 (COVID-19) patients are unknown. This study aimed to describe the clinical implications of preexisting neurological comorbidities and new-onset neurological complications in ICU patients with COVID-19. ICU patients who were isolated and treated for COVID-19 between 19 February 2020 and 3 May 2020, from one tertiary hospital and one government-designated branch hospital were included. Clinical data including previous neurological disorders were extracted from electronic medical records. All neurological complications were evaluated by neurointensivists. Multiple logistic regression analysis was performed to investigate independent factors in ICU mortality. The median age of 52 ICU patients with COVID-19 was 73 years. Nineteen (36.5\%) patients had preexisting neurological comorbidities, and new-onset neurological complications occurred in $23(44.2 \%)$ during ICU admission. Patients with preexisting neurological comorbidities required tracheostomy more frequently and more ventilator and ICU days than those without. Patients with new-onset neurological complications experienced more medical complications and had higher ICU severity score and ICU mortality rates. New-onset neurological complications remained an independent factor for ICU mortality. Many COVID-19 patients in the ICU have preexisting neurological comorbidities, making them at a high risk of new-onset neurological complications.
\end{abstract}

Keywords: coronavirus; neurology; critical care; neurocritical care; mortality

\section{Introduction}

The novel coronavirus disease (COVID-19) outbreak in Wuhan, China, has resulted in an ongoing pandemic and is associated with high morbidity and mortality, particularly in conjunction with old age and some underlying medical conditions [1-3]. Recent reports from the Centers for Disease Control and Prevention (CDC) in the United States have indicated that cancer, chronic kidney disease, heart failure, coronary artery disease, and type 2 diabetes mellitus, for example, put patients at an increased risk for severe illness from COVID-19 [4]. However, the clinical effects of underlying neurological conditions remain uncertain because of a lack of evidence. 
COVID-19 can lead to various neurological complications and lethal respiratory infections such as acute respiratory distress syndrome (ARDS) [1]. Neurological manifestations were found in $36.4 \%$ of COVID-19 patients admitted to the hospital in Wuhan, China, which were more often found in more severe disease [5]. Serious neurological complications, including acute stroke and impaired consciousness, were relatively common $[5,6]$. In addition, data from the two intensive care units (ICUs) for COVID-19 patients in France showed that agitation and delirium were observed in approximately two-thirds of ICU patients, and neurological findings were observed in $67 \%$ of patients when sedation and neuromuscular blockers were discontinued [7]. However, little is known about how new-onset neurological complications affect severe COVID-19 patients.

Herein, we aimed to investigate the clinical implications of preexisting neurological comorbidities and new-onset neurological complications in COVID-19 patients admitted to the ICU.

\section{Materials and Methods}

\subsection{Selection of Study Subjects}

The city of Daegu emerged as the epicenter of COVID-19 in South Korea and has accounted for more than two-thirds of the over 10,000 infections in the country. A total of 1004 patients were isolated and treated for COVID-19 from 19 February 2020 to 3 May 2020, in two hospitals in Daegu: Keimyung University Dongsan Hospital (a tertiary hospital) and Daegu Dongsan Hospital (a government-designated branch hospital). The governmentdesignated branch hospital served as a regional hub to exclusively treat COVID-19 patients. As the number of new cases of COVID-19 decreased rapidly in Daegu, the ICU of the branch hospital was closed on 3 May 2020, which allowed us to obtain the final medical outcomes from most of the patients.

All subjects were tested for severe acute respiratory syndrome coronavirus 2 (SARS$\mathrm{CoV}-2$ ) using a real-time reverse transcription-polymerase chain reaction (RT-PCR) assay of a nasal swab specimen based on the recommendations of the CDC and the Korean CDC $[8,9]$. Patients who were admitted to the integrated ICU for critical care were enrolled in our study. A collaborative approach was designed and implemented by our critical care team, and various subspecialized intensivists were dispatched by the Korean Society of Critical Care Medicine.

\subsection{Ethical Considerations}

Our study was approved by the local institutional review boards (IRBs; IRB No. 202005-048) of the hospitals for the collection of anonymized clinical data with a waiver of informed consent because of the study's retrospective design, subject anonymity, and minimal risk to participants. Patients' clinical characteristics, laboratory parameters, treatments, and outcomes were obtained from the Dongsan COVID-19 registry. This study was conducted in accordance with the Declaration of Helsinki.

\subsection{Data Collection and Definitions}

The criteria for ICU admission included clinical conditions in which intensive care and continuous monitoring were required because of severe symptoms accompanied by multiple organ failure after a positive COVID-19 diagnosis. Specific conditions included severe pneumonia (respiration rate $>30$ or $<90 \%$ oxygen saturation with dyspnea), ARDS, and sepsis (with or without shock).

Specific signs and symptoms of COVID-19 were collected from questionnaires taken at the time of hospitalization and included fever, chills, cough, sputum production, rhinorrhea, sore throat, myalgia or fatigue, headache, diarrhea, dyspnea, and chest pain. At the early period of the COVID-19 crisis, new-onset loss of taste or smell was not a well-known symptom; therefore, it was not assessed in all ICU patients.

Preexisting neurological comorbidities were ascertained through self-reporting, medication review, and hospital record review. Preexisting neurological comorbidities were 
divided into (1) neurodegenerative diseases, (2) cerebrovascular diseases, and (3) other neurological diseases. Neurodegenerative diseases included Alzheimer's disease and other dementias, Parkinson's disease (PD) and PD-related disorders, and motor neuron diseases. Cerebrovascular diseases were defined as any disorder in which an area of the brain was temporarily or permanently affected by ischemia or bleeding. The categories in "other neurological diseases" included all other neurological disorders except neurodegenerative and cerebrovascular diseases.

Clinical complications included new-onset neurological complications, ARDS, septic shock, and acute kidney injury (AKI). All these complications that occurred from the ICU admission day were collected. New-onset neurological complications were seizure, acute stroke, diffuse hypoxic brain injury, and delirium. Seizure was diagnosed as a clinical seizure or electrical seizure activity in the electroencephalogram (EEG) without any clinical signs of seizure (non-convulsive seizure) if COVID-19 patients had unexplained mental changes. Acute stroke was defined as an acute neurological deficit with focal signs and symptoms upon neurological examination, regardless of symptom duration, and was classified as ischemic or hemorrhagic based on computed tomography (CT) or magnetic resonance imaging (MRI). Delirium was diagnosed by intensivists using the Intensive Care Delirium Screening Checklist or Confusion Assessment Method for the ICU. All newonset neurological complications during ICU treatment were evaluated and confirmed by neurointensivists. Seizure and acute stroke were treated by each guideline. Principles of delirium management were supportive medical care and treatment of underlying conditions. If indicated, antipsychotics such as quetiapine or haloperidol were administered for severe agitation [10].

The definition of ARDS was based on the World Health Organization (WHO) guidelines for COVID-19 [11]. Briefly, ARDS was defined as a $\mathrm{PaO}_{2} / \mathrm{FiO}_{2}$ ratio $<300 \mathrm{mmHg}$ with a positive end-expiratory pressure $\geq 5 \mathrm{~cm} \mathrm{H}_{2} \mathrm{O}$ within 1 week of known clinical insult, or new or worsening respiratory symptoms. Septic shock was defined as persistent hypotension despite volume resuscitation requiring vasopressors to maintain a mean arterial pressure $\geq 65 \mathrm{mmHg}$ and a serum lactate level $>2 \mathrm{mmol} / \mathrm{L}$. AKI was defined using the Kidney Disease Improving Global Outcome criteria [12]. AKI was diagnosed if any one of the following was present: (1) a serum creatinine increase of $\geq 0.3 \mathrm{mg} / \mathrm{dl}$ within $48 \mathrm{~h}$, (2) a serum creatinine increase of $\geq 1.5$ times the baseline within 7 days, or (3) a urine volume $<0.5 \mathrm{~mL} / \mathrm{kg} / \mathrm{h}$ for $6 \mathrm{~h}$.

The Sequential Organ Failure Assessment (SOFA) score reflects organ failure/dysfunction over time, which can predict mortality in critically ill patients [13]. We used the patients' worst recorded values $\left(\mathrm{PaO}_{2} / \mathrm{FiO}_{2}\right.$ ratio, platelet count, bilirubin, mean arterial pressure with inotropes, Glasgow Coma Score, and creatinine or urine output) within $24 \mathrm{~h}$ of ICU admission to calculate SOFA scores and evaluate baseline severity. Acute Physiology and Chronic Health Evaluation (APACHE) II score within $24 \mathrm{~h}$ of ICU admission with worst value recorded for each component part of 12 physiology variables was also employed [14]

Time variables were calculated with respect to length of ICU stay, ventilator days, and symptom onset-to-ICU admission. If a patient no longer required critical care and intensive monitoring in the ICU, as determined by intensivists, they were discharged or transferred from the ICU regardless of the RT-PCR results. The rate of transfer out of the ICU and ICU death were reported as clinical outcomes.

\subsection{Statistical Analysis}

Categorical variables are presented as counts and percentages and continuous variables as median and interquartile range (IQR) because of the small number of subjects. To compare the baseline characteristics according to the existence of neurological comorbidities or complications, the Pearson $\chi^{2}$-test or Fisher's exact test was used for categorical variables and Mann-Whitney $U$ test was used for continuous variables, because of the number of subjects assigned to each group. Significance levels were set at $p<0.05$ for twotailed tests. Cumulative risks of new-onset neurological complications in the two groups 
according to preexisting neurological comorbidities were calculated using Kaplan-Meier estimates. The Cox proportional hazard model was applied to evaluate the influences on new-onset neurological complications according to preexisting neurological comorbidities. Multiple logistic regression analysis was used to evaluate the independent predictors of ICU mortality in ICU patients with COVID-19. Predefined variables (age, sex, and APACHE II score) were adjusted in both of the statistical analysis models because of an overfitting issue. All statistical analyses were performed using SPSS (version 19.0; SPSS Inc., Chicago, IL, USA).

\section{Results}

Of the 1004 patients infected with COVID-19 admitted to the integrated ICU for critical care from the two hospitals, 52 were included in the study, including 15 of 40 patients from the tertiary hospital and 37 of 964 from the government-designated branch hospital. The median age of our patient cohort was 73 (IQR 57-81) years, and 51.9\% were male. Patients with preexisting neurological comorbidities $(79(59-84)$ vs. $68(56-78)$ years, $p=0.114$ ) or new-onset neurological complications (79 (63-85) vs. 68 (54-77) years, $p=0.054)$ tended to be old. The proportion of male patients with preexisting neurological comorbidities was higher than those without $(73.7 \%$ vs. $39.4 \%, p=0.017)$. At baseline, no significant differences were found in the proportion of underlying medical conditions between the preexisting neurological comorbidity and new-onset neurological complication groups. The median APACHE II and SOFA scores at admission were 11 (7-21) and 4 (2-6), respectively. Although no significant difference was observed in the APACHE II and SOFA score between the two groups according to preexisting neurological comorbidities (12 (9-21) vs. 10 (7-20), $p=0.232 ; 4(2-7)$ vs. $3(2-5), p=0.343)$, patients with new-onset neurological complications had significantly higher APACHE II and SOFA scores than those without (12 (9-22) vs. 9 (7-19), $p=0.040 ; 5(2-7)$ vs. $2(1-5), p=0.044)$. Among 52 ICU patients, $25 \%$ did not complain of COVID-19-related signs or symptoms at initial admission. Patients with preexisting neurological comorbidities had significantly lower rates of sore throat ( $0 \% \mathrm{vs}$. $27.3 \%, p=0.018)$, myalgia or fatigue $(10.5 \%$ vs. $54.5 \%, p=0.002)$, and headache $(5.3 \%$ vs. $33.3 \%, p=0.037$ ) than those without (Table 1).

Table 2 shows preexisting neurological comorbidities and new-onset neurological and medical complications. Preexisting neurological comorbidities existed in 19 (36.5\%) patients, including seven patients with neurodegenerative diseases (four with Alzheimer's disease or other dementias, two with PD and PD-related disorders, and two with motor neuron disease), seven patients with cerebrovascular diseases, and five patients with other neurological diseases, all of which were present before ICU admission. Two preexisting neurological comorbidities overlapped in one patient (PD with dementia). The five cases of other neurological diseases included one case of spasmodic dystonia, one case of encephalitis, two cases of epilepsy, and one case of traumatic brain injury.

As regards medical complications, ARDS, AKI, and septic shock occurred in $40.4 \%$, $25 \%$, and $42.3 \%$ of the patients, respectively. A total of $23(44.2 \%)$ patients had newonset neurological complications, three (5.8\%) had acute stroke, three $(5.8 \%)$ experienced seizures, and $20(38.5 \%)$ had delirium. The median onset time of new-onset neurological complications was 8 (2-10) days after ICU admission: seizure, 8 (10-13) days; acute stroke, 11 (1-19) days; and delirium $2(2-8)$ days. The three cases of acute stroke were all classified as ischemic. ARDS (60.9\% vs. $24.2 \%, p=0.017)$ and septic shock $(60.9 \%$ vs. $27.6 \%, p=0.033)$ occurred more frequently in patients with new-onset neurological complications than in those without.

Moreover, $50 \%$ of patients were treated with a mechanical ventilator; extracorporeal membrane oxygenation (ECMO) and continuous renal replacement therapy (CRRT) were applied in $9.6 \%$ and $15.4 \%$, respectively. Patients with preexisting neurological comorbidities underwent tracheostomy more frequently ( $36.8 \%$ vs. $6.1 \%, p=0.008)$. The durations (days) of invasive ventilation ( 23 vs. $5, p=0.013$ ) and ICU stay (27 vs. $16, p=0.048$ ) were longer in patients with preexisting neurological comorbidities than in those with- 
out. Mechanical ventilation was performed more frequently on patients with new-onset neurological complications (69.6\% vs. $34.5 \%, p=0.026)$ (Table 3 ).

Of the 19 patients with preexisting neurological comorbidities, 14 (73.7\%) had newonset neurological complications, which was significantly higher than that in the group without neurological complications $(73.7 \%$ vs. $27.3 \%, p=0.001)$. Figure 1 shows the KaplanMeier curve of the cumulative risk of new-onset neurological complications according to preexisting neurological comorbidities. The Cox proportional hazards model showed that preexisting neurological comorbidities was an independent predictor of future newonset neurological complications (hazard ratio [HR], 2.894; 95\% confidence interval [CI], $1.194-7.011, p=0.019)$.

Table 1. Baseline characteristics of 52 intensive care unit patients infected with COVID-19.

\begin{tabular}{|c|c|c|c|c|c|c|c|}
\hline & $\begin{array}{l}\text { All ICU } \\
\text { Patients } \\
\mathbf{N}=52\end{array}$ & $\begin{array}{c}\text { Preexisting } \\
\text { Neurological } \\
\text { Comorbidities } \\
\mathrm{N}=19(36.5 \%)\end{array}$ & $\begin{array}{c}\text { No } \\
\text { Preexisting } \\
\text { Neurological } \\
\text { Comorbidities } \\
\mathrm{N}=33(63.5 \%)\end{array}$ & $p$ Value & $\begin{array}{c}\text { New-Onset } \\
\text { Neurological } \\
\text { Complications } \\
\mathrm{N}=23(44.2 \%)\end{array}$ & $\begin{array}{c}\text { No } \\
\text { New-Onset } \\
\text { Neurological } \\
\text { Complications } \\
\mathrm{N}=\mathbf{2 9}(\mathbf{4 5 . 8 \% )}\end{array}$ & $p$ Value \\
\hline \multicolumn{8}{|l|}{ Characteristics } \\
\hline Age, median (IQR), year & $73(57-81)$ & $79(59-84)$ & $68(56-78)$ & $0.114^{+}$ & $79(63-85)$ & $68(54-77)$ & $0.054^{\dagger}$ \\
\hline Sex, male $(\%)$ & $27(51.9)$ & $14(73.7)$ & $13(39.4)$ & $0.017 \int$ & $13(56.5)$ & $14(48.3)$ & $0.755 \int$ \\
\hline \multicolumn{8}{|l|}{$\begin{array}{l}\text { Underlying medical } \\
\text { conditions }(\%)\end{array}$} \\
\hline Hypertension & $29(55.8)$ & $10(52.6)$ & $19(57.6)$ & $0.730 \int$ & $14(60.9)$ & $15(51.7)$ & $0.705 \int$ \\
\hline Diabetes mellitus & $24(46.2)$ & $7(36.8)$ & $17(51.5)$ & $0.307 \int$ & $9(39.1)$ & $15(51.7)$ & $0.532 \int$ \\
\hline Dyslipidemia & $5(9.5)$ & $2(10.5)$ & $3(9.1)$ & $1 *$ & $4(17.4)$ & $1(3.4)$ & 0.222 * \\
\hline Cardiac disease & $9(17.3)$ & $5(26.3)$ & $4(12.1)$ & $0.260 *$ & $6(26.1)$ & $3(10.3)$ & $0.262 *$ \\
\hline Malignancy & $6(11.5)$ & $2(10.5)$ & $4(12.1)$ & $1 *$ & $4(17.4)$ & $2(6.9)$ & $0.460 *$ \\
\hline Chronic liver disease & $2(3.8)$ & $1(5.3)$ & $1(3)$ & $1 *$ & $2(8.7)$ & $0(0.0)$ & 0.372 * \\
\hline Chronic kidney disease & $6(11.5)$ & $4(21.1)$ & $2(6.1)$ & $0.175^{*}$ & $5(21.7)$ & $1(3.4)$ & 0.107 * \\
\hline \multicolumn{8}{|l|}{$\begin{array}{l}\text { Scoring system, median } \\
\text { (IQR) }\end{array}$} \\
\hline SOFA score & $4(2-6)$ & $4(2-7)$ & $3(2-5)$ & $0.343^{\dagger}$ & $5(2-7)$ & $2(1-5)$ & $0.044^{+}$ \\
\hline APACHE II score & $11(7-21)$ & $12(9-21)$ & $10(7-20)$ & $0.232^{+}$ & $12(9-22)$ & $9(7-19)$ & $0.040^{+}$ \\
\hline Signs and symptoms (\%) & $39(75.0)$ & $13(68.4)$ & $26(78.8)$ & $0.406 \int$ & $15(65.2)$ & $24(82.8)$ & $0.147 \int$ \\
\hline Fever & $35(67.3)$ & $12(63.2)$ & $23(69.7)$ & $0.628^{\int}$ & $13(56.5)$ & $22(75.9)$ & $0.238^{\int}$ \\
\hline Chilling & $10(19.2)$ & $2(10.5)$ & $8(24.2)$ & 0.293 * & $4(17.4)$ & $6(20.7)$ & $1 *$ \\
\hline Cough & $22(42.3)$ & $5(26.3)$ & $17(51.5)$ & $0.077 \int$ & $7(30.4)$ & $15(51.7)$ & $0.207 \int$ \\
\hline Sputum production & $15(28.8)$ & $6(31.6)$ & $9(27.3)$ & $0.741 \int$ & $5(21.7)$ & $10(34.5)$ & $0.484 \int$ \\
\hline Rhinorrhea & $6(11.5)$ & $2(10.5)$ & $4(12.1)$ & $1 *$ & $2(8.7)$ & $4(13.8)$ & 0.893 * \\
\hline Sore throat & $9(17.3)$ & $0(0)$ & $9(27.3)$ & $0.018 *$ & $3(13.0)$ & $6(20.7)$ & 0.723 * \\
\hline Myalgia or fatigue & $20(38.5)$ & $2(10.5)$ & $18(54.5)$ & $0.002 \int$ & $6(26.1)$ & $14(48.3)$ & $0.178^{\int}$ \\
\hline Headache & $12(23.1)$ & $1(5.3)$ & $11(33.3)$ & $0.037 *$ & $6(26.1)$ & $6(20.7)$ & $0.646 \int$ \\
\hline Diarrhea & $10(19.2)$ & $3(15.8)$ & $7(21.2)$ & $0.729 *$ & $4(17.4)$ & $6(20.7)$ & 1.000 * \\
\hline Dyspnea & $21(40.4)$ & $5(26.3)$ & $16(48.5)$ & $0.117 \int$ & $8(34.8)$ & $13(44.8)$ & $0.654 \int$ \\
\hline Chest pain & $1(1.9)$ & $1(5.3)$ & $0(0)$ & $0.365 *$ & $1(4.3)$ & $0(0)$ & 0.907 * \\
\hline
\end{tabular}

COVID-19, coronavirus disease 2019; IQR, interquartile range; SOFA, Sequential Organ Failure Assessment; APACHE, Acute Physiology and Chronic Health Evaluation. Data are median (interquartile range), $\mathrm{n}(\%)$, or $\mathrm{n} / \mathrm{N}(\%)$, where $\mathrm{N}$ is the total number of patients with available data. $p$ values were obtained using $\chi^{2}$ test $\int$, Fisher' exact test *, or Mann-Whitney $\mathrm{U}^{*}$ test $^{\dagger}$.

HR, hazard ratio; CI, confidence interval. Among the 52 patients, the ICU mortality rate was $33.3 \%$. Patients with new-onset neurological complications had a significantly higher rate of ICU mortality than those without (56.5\% vs. $20.7 \%, p=0.008)$ (Table 3 ). After adjusting for age, sex, and APACHE II score, new-onset neurological complications (odds ratio [OR], 6.18; 95\% CI, 1.16-32.90, $p=0.03$ ) and APACHE II score (OR, 1.20; 95\% CI, 1.06-1.36, $p=0.003$ ) remained independent predictors of ICU mortality (Table 4). No differences in the results were observed when the SOFA score was included in the analysis instead of the APACHE II score (Table S1). Patients with delirium had a significantly higher rate of preexisting neurological comorbidities $(65.0 \%$ vs. $18.8 \%, p=0.001)$, chronic kidney disease $(25.0 \%$ vs. $3.1 \%, p=0.026)$, and ICU mortality than those without ( $55.0 \%$ vs. $25.0 \%$, $p=0.029$ ) (Table S2). 
Table 2. Preexisting neurological comorbidities and new-onset neurological complications of 52 intensive care unit patients infected with COVID-19.

\begin{tabular}{|c|c|c|c|c|c|c|c|}
\hline & $\begin{array}{l}\text { All ICU } \\
\text { Patients } \\
\mathrm{N}=52\end{array}$ & $\begin{array}{c}\text { Preexisting } \\
\text { Neurological } \\
\text { Comorbidities } \\
\mathbf{N}=19(36.5 \%)\end{array}$ & $\begin{array}{c}\text { No } \\
\text { Preexisting } \\
\text { Neurological } \\
\text { Comorbidities } \\
\mathrm{N}=33(63.5 \%)\end{array}$ & $p$ Value & $\begin{array}{l}\text { New-Onset } \\
\text { Neurological } \\
\text { Complications } \\
\mathrm{N}=23(44.2 \%)\end{array}$ & $\begin{array}{c}\text { No } \\
\text { New-Onset } \\
\text { Neurological } \\
\text { Complications } \\
\mathrm{N}=29(45.8 \%)\end{array}$ & $p$ Value \\
\hline \multicolumn{8}{|l|}{ Characteristics } \\
\hline $\begin{array}{l}\text { Preexisting neurologic } \\
\text { comorbidities }(\%)\end{array}$ & $19(36.5)$ & $19(100)$ & & & $14(60.9)$ & 5 (17.2) & $0.0011^{\int}$ \\
\hline Neurodegenerative disease & $7(13.5)$ & $7(36.8)$ & & & $5(21.7)$ & $2(6.9)$ & 0.219 * \\
\hline $\begin{array}{l}\text { Alzheimer disease and } \\
\text { other dementias }\end{array}$ & $4(7.7)$ & $4(21.1)$ & & & $4(17.4)$ & $0(0)$ & $0.033 *$ \\
\hline $\begin{array}{l}\text { PD and PD-related } \\
\text { disorders }\end{array}$ & $2(3.8)$ & $2(10.5)$ & & & $1(4.3)$ & $1(3.4)$ & $1.000 *$ \\
\hline $\begin{array}{l}\text { Motor neuron disease } \\
\text { Cerebrovascular disease } \\
\text { Other neurologic disease } \\
\text { Complications (\%) }\end{array}$ & $\begin{array}{c}2(3.8) \\
7(13.5) \\
5(9.6)\end{array}$ & $\begin{array}{l}2(10.5) \\
7(36.8) \\
5(26.3)\end{array}$ & & & $\begin{array}{l}1(4.3) \\
5(21.7) \\
4(17.4)\end{array}$ & $\begin{array}{l}1(3.4) \\
2(6.9) \\
1(3.4)\end{array}$ & $\begin{array}{l}1.000 * \\
0.219 * \\
0.157 *\end{array}$ \\
\hline Neurological complications & $23(44.2)$ & $14(73.7)$ & $9(27.3)$ & $0.001 \int$ & $23(100)$ & & \\
\hline Seizure & $3(5.8)$ & $2(10.5)$ & $1(3.0)$ & $0.546 *$ & $3(13.0)$ & & \\
\hline Acute stroke & $3(5.8)$ & $3(15.8)$ & $0(0)$ & 0.044 * & $3(13.0)$ & & \\
\hline Delirium & $20(38.5)$ & $13(68.4)$ & $7(21.2)$ & $0.001 \int$ & $20(87.0)$ & & \\
\hline $\begin{array}{l}\text { Acute respiratory distress } \\
\text { syndrome }\end{array}$ & $21(40.4)$ & $9(47.4)$ & $12(36.4)$ & $0.436 \int$ & $14(60.9)$ & $7(24.1)$ & $0.017 \int$ \\
\hline Acute kidney injury & $13(25)$ & $6(31.6)$ & $7(21.2)$ & 0.510 * & $7(30.4)$ & $6(20.7)$ & $0.629 \int$ \\
\hline Septic shock & $22(42.3)$ & $10(52.6)$ & $12(36.4)$ & $0.253 \int$ & $14(60.9)$ & $8(27.6)$ & $0.033 \int$ \\
\hline
\end{tabular}

COVID-19, coronavirus disease 2019; IQR, interquartile range; PD, Parkinson's disease. Data are median (interquartile range), $\mathrm{n}(\%)$, or $\mathrm{n} / \mathrm{N}(\%)$, where $\mathrm{N}$ is the total number of patients with available data. $p$ values were obtained using $\chi^{2} \int$ or Fisher' exact test*.

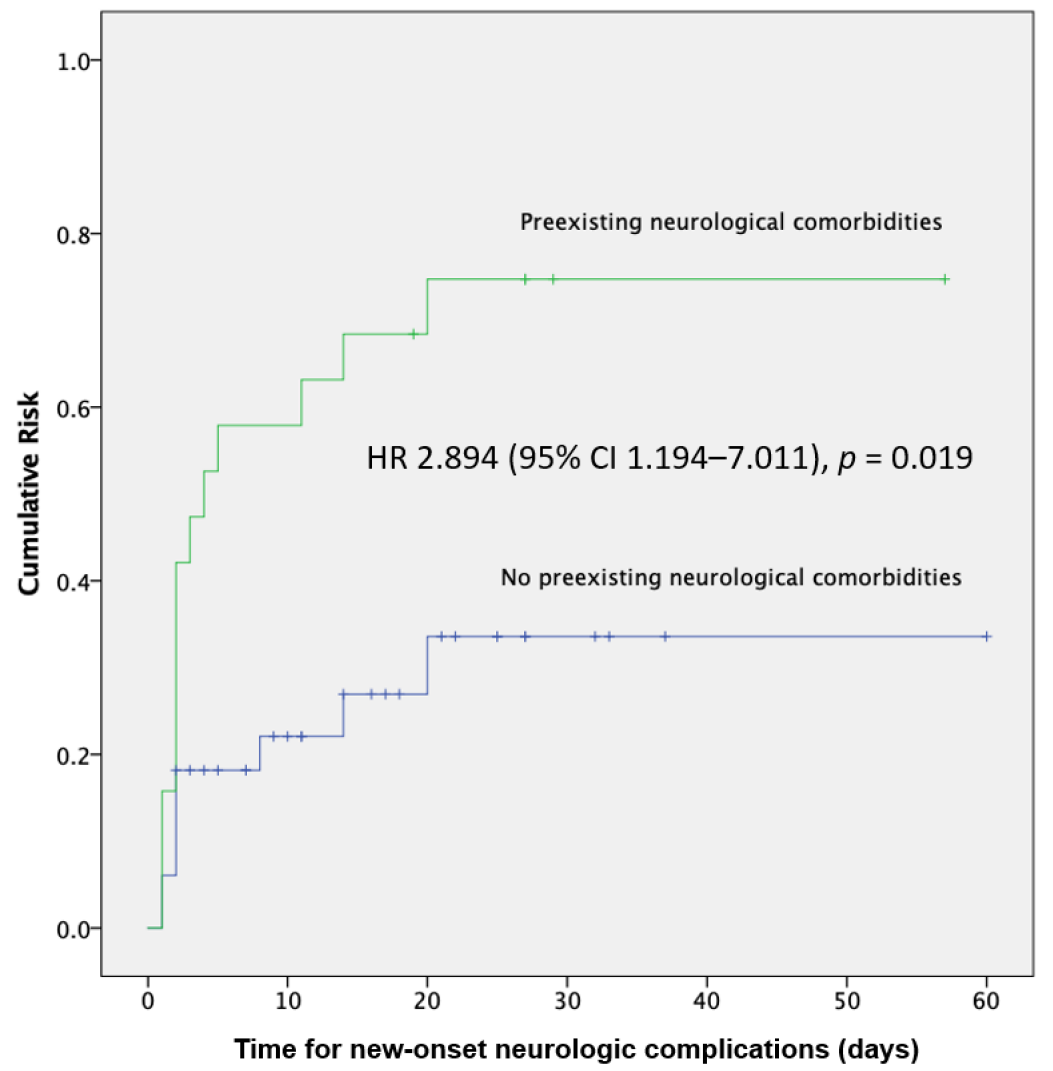

Figure 1. Kaplan-Meier estimates of the cumulative risk of new-onset neurological complications. 
Table 3. Treatment and prognosis of 52 intensive care unit patients infected with COVID-19.

\begin{tabular}{|c|c|c|c|c|c|c|c|}
\hline & $\begin{array}{l}\text { All ICU } \\
\text { Patients } \\
\mathrm{N}=52\end{array}$ & $\begin{array}{c}\text { Preexisting } \\
\text { Neurological } \\
\text { Comorbidities } \\
\mathrm{N}=19(36.5 \%)\end{array}$ & $\begin{array}{c}\text { No } \\
\text { Preexisting } \\
\text { Neurological } \\
\text { Comorbidities } \\
\mathrm{N}=33(63.5 \%)\end{array}$ & $p$ Value & $\begin{array}{c}\text { New-Onset } \\
\text { Neurological } \\
\text { Complications } \\
\mathbf{N}=23(44.2 \%)\end{array}$ & $\begin{array}{c}\text { No } \\
\text { New-Onset } \\
\text { Neurological } \\
\text { Complications } \\
\mathrm{N}=29(45.8 \%)\end{array}$ & $p$ Value \\
\hline \multicolumn{8}{|l|}{ Treatments (\%) } \\
\hline \multirow{3}{*}{$\begin{array}{l}\text { Mechanical ventilation } \\
\text { non-invasive facial } \\
\text { mask } \\
\text { invasive }\end{array}$} & $26(50)$ & $11(57.9)$ & $15(45.5)$ & $0.388^{\int}$ & $16(69.6)$ & $10(34.5)$ & $0.026^{\int}$ \\
\hline & $9(17.3)$ & $3(15.8)$ & $6(18.2)$ & $1^{*}$ & $4(17.4)$ & $5(17.2)$ & $1^{*}$ \\
\hline & $23(44.2)$ & $10(52.6)$ & $13(39.4)$ & $0.355^{\delta}$ & $15(65.2)$ & $8(27.6)$ & $0.015^{\int}$ \\
\hline $\begin{array}{l}\text { Extracorporeal membrane } \\
\text { oxygenation }\end{array}$ & $5(9.6)$ & $3(15.8)$ & $2(6.1)$ & $0.342 *$ & $4(17.4)$ & $1(3.4)$ & $0.222 *$ \\
\hline $\begin{array}{l}\text { Continuous renal } \\
\text { replacement therapy }\end{array}$ & $8(15.4)$ & $5(26.3)$ & $3(9.1)$ & 0.124 * & $6(26.1)$ & $2(6.9)$ & $0.129 *$ \\
\hline $\begin{array}{l}\text { Antibiotic treatment } \\
\text { Antiviral treatment }\end{array}$ & $\begin{array}{l}49(94.2) \\
49(94.2)\end{array}$ & $\begin{array}{l}18(94.7) \\
17(89.5)\end{array}$ & $\begin{array}{l}31(93.9) \\
32(97)\end{array}$ & $\begin{array}{c}1 * \\
0.546^{*}\end{array}$ & $\begin{array}{l}23(100) \\
23(100)\end{array}$ & $\begin{array}{l}26(89.7) \\
26(89.7)\end{array}$ & $\begin{array}{l}0.245 * \\
0.245^{*}\end{array}$ \\
\hline Glucocorticoids & $39(75)$ & $14(73.7)$ & $25(75.8)$ & $1 *$ & $20(87.0)$ & $19(65.5)$ & $0.147 \int$ \\
\hline $\begin{array}{l}\text { Intravenous } \\
\text { immunoglobulin therapy }\end{array}$ & $1(1.9)$ & $0(0)$ & $1(3)$ & $1 *$ & $0(0)$ & $1(3.4)$ & $1 *$ \\
\hline \multicolumn{8}{|c|}{$\begin{array}{l}\text { Tracheostomy } \\
\text { Treatment Timeline, median (IQR), } \\
\text { days-n/N. }\end{array}$} \\
\hline \multirow{3}{*}{$\begin{array}{l}\text { length of ICU stays } \\
\text { Invasive ventilator days, } \\
23 / 52 \\
\text { Onset-to-ICU admission, } \\
39 / 52 \\
\text { Prognosis-n/N. (\%) }\end{array}$} & $22(9-32)$ & $27(18-32)$ & $16(7-30)$ & $0.048^{+}$ & $26(8-38)$ & 19 (10-27) & $0.361^{\dagger}$ \\
\hline & $18(3-29)$ & $\begin{array}{c}23 \\
(18-37) 10 / 19\end{array}$ & $5(2-20) 13 / 33$ & $0.013^{\dagger}$ & $18(5-30) 15 / 23$ & $7(2-28) 8 / 29$ & $0.437^{\dagger}$ \\
\hline & $8(6-11)$ & $8(7-10) 13 / 19$ & $9(5-11) 26 / 33$ & $0.419^{\dagger}$ & $8(7-11) 15 / 23$ & $8(5-11) 24 / 29$ & $0.674^{+}$ \\
\hline \multirow[t]{2}{*}{ Transfer out of ICU } & $\begin{array}{l}33 / 52 \\
(63.5)\end{array}$ & $10 / 19(52.6)$ & 23/33 (69.7) & \multirow[t]{2}{*}{$0.2188^{\int}$} & $10 / 23(43.5)$ & $23 / 29(79.3)$ & \multirow[t]{2}{*}{$0.008 \int$} \\
\hline & $\begin{array}{l}16 / 48 \\
(33.3)\end{array}$ & 9/19 (47.4) & $10 / 33(30.3)$ & & $13 / 23(56.5)$ & $6 / 29(20.7)$ & \\
\hline
\end{tabular}

COVID-19, coronavirus disease 2019; ICU, intensive care unit; IQR, interquartile range. Data are median (interquartile range), $\mathrm{n}$ (\%), or $\mathrm{n} / \mathrm{N}(\%)$, where $\mathrm{N}$ is the total number of patients with available data. $p$ values were obtained using $\chi^{2}$ test $\int$, Fisher' exact test *, or Mann-Whitney U test ${ }^{\dagger}$.

Table 4. Independent predictors associated with ICU mortality in the multiple logistic regression analysis using APACHE II score.

\begin{tabular}{lccc}
\hline \multicolumn{1}{c}{ Model 1 } & OR & $\mathbf{9 5 \%}$ C.I. & $p$-Value \\
\hline Age & 1.01 & $0.95-1.07$ & 0.81 \\
Male & 4.15 & $0.79-21.84$ & 0.09 \\
APACHE II score & 1.20 & $1.06-1.36$ & 0.003 \\
New-onset neurologic & 6.18 & $1.16-32.90$ & 0.03 \\
complications & & & \\
\hline
\end{tabular}

Nagelkerke $R^{2}=0.576$

Hosmer-Lemeshow Chi-square test $=7.955, d f=8, p$-value $=0.438$

\begin{tabular}{lccc}
\hline \multicolumn{1}{c}{ Model 2 } & OR & $\mathbf{9 5 \%}$ C.I. & $p$-Value \\
\hline Age & 1.01 & $0.95-1.08$ & 0.73 \\
Male & 5.23 & $0.82-33.23$ & 0.08 \\
APACHE II score & 1.21 & $1.06-1.37$ & 0.004 \\
$\begin{array}{l}\text { Preexisting } \\
\text { neurological }\end{array}$ & 0.55 & $0.77-3.88$ & 0.55 \\
$\begin{array}{l}\text { comorbidities } \\
\text { New-onset neurologic }\end{array}$ & 7.86 & $1.21-51.32$ & 0.03 \\
complications & & & \\
\hline
\end{tabular}

Nagelkerke $R^{2}=0.581$

Hosmer-Lemeshow Chi-square test $=4.141, d f=8, p$-value $=0.844$

CI, confidence interval; ICU, intensive care unit; OR, odds ratio; APACHE, Acute Physiology and Chronic Health Evaluation. 


\section{Discussion}

To the best of our knowledge, this is the first study on the clinical implications of preexisting neurological comorbidities and new-onset neurological complications in ICU patients infected with COVID-19.

First, we focused on the effect of COVID-19 on ICU patients with preexisting neurological disease. Recently, García-Azorín et al. from Spain evaluated the association between the prognosis and comorbid chronic neurological disorders in COVID-19 patients and found that preexisting chronic neurological disorders were independent predictors of mortality in hospitalized COVID-19 patients [15]. Another study from Wuhan, China, reported that COVID-19 patients with preexisting stroke were readily predisposed to death (mortality between patients with and without stroke, 45\% vs. 9\%, adjusted HR 1.73 [95\% CI, 1.00-2.98]) [16]. These studies were based on all hospitalized COVID-19 patients. Our study only enrolled patients admitted to the ICU who, compared with the patients of the abovementioned referenced studies, had a more severe condition based on the median SOFA score (4 in our study vs. 1 in the Chinese study) and mortality rate (33\% in our study vs. $22 \%$ in the Spanish study vs. $12 \%$ in the Chinese study). In our study, preexisting neurological comorbidities increased the duration of ventilator use, length of ICU stay, and need for a tracheostomy. Those with neurological conditions are at an increased risk of ICU-acquired and ventilator-associated pneumonia because of impaired airway reflexes, including difficulties in swallowing and coughing [17,18]. Patients with preexisting neurological comorbidities had a significantly lower rate of subjective COVID-19-related symptoms, such as sore throat, myalgia, fatigue, or headache before admission; however, it is likely that patients with preexisting neurological comorbidities were not capable of self-reporting such symptoms. Cough symptoms were also observed at a relatively low rate in patients who already had neurological disorders, including stroke, head trauma, PD, and motor neuron disease, all of which may have reduced the cough reflexes. Therefore, a thorough patient history, including preexisting neurological comorbidities, and adequate airway management in patients with preexisting neurological comorbidities are important when addressing COVID-19-related symptoms. However, this did not affect the mortality rate of ICU patients with COVID-19. This is because both groups were already in a severe condition when they were admitted to the ICU, and the multidisciplinary team, including neurointensivists, involved in all care had a positive effect on the mortality rate [19].

This study also examined the effects of new-onset neurological complications in ICU patients with COVID-19 in relation to the clinical effect, including ICU mortality. Cases of direct involvement of SARS-CoV-2 into the central or peripheral nervous system are very rare [20]. However, recent evidence has emerged regarding a post-viral autoimmune phenomenon including indirect involvement [21-24]. Mao et al., from China, recently reported that one-third of COVID-19 patients have neurological symptoms, which are more common (45.5\%) in patients with severe infections. In our study, new-onset neurological complications occurred in approximately $44 \%$ of patients who were treated for COVID-19 in the ICU. Severe neurological complications, such as acute cerebrovascular disease and consciousness disturbance, occurred more frequently in the later stages of the disease [5]. In our study, seizure and acute stroke occurred within $>7$ days of ICU admission.

Seizure has not been widely reported in patients with COVID-19. SARS-CoV-2 infection may cause multiple organ failures, metabolic derangements, and hypoxia, which can lead to clinical or subclinical seizures [25]. Acute cerebrovascular disease is considered a major cause of seizure. Acute stroke is an emerging COVID-19 complication, with cohort studies reporting stroke in $2 \%-6 \%$ of patients, similar to the rate seen in our study (5.8\%) [26]. The link between SARS-CoV-2 and cerebrovascular disease is an important mechanism for new-onset neurological complications. SARS-CoV-2-induced inflammatory responses result in coagulation and thrombin generation and are followed by thromboembolism and D-dimer accumulation that can influence the onset or worsening of stroke [27-29]. ARDS, acute cardiac injury, and septic shock have been reported to occur frequently in ICU patients with COVID-19 [30,31]. Neurological complications may 
be caused by COVID-19-related medical complications [7,32]. Delirium in the ICU has a negative effect on the outcome of patients [32,33].

Preexisting neurological comorbidities can affect new-onset neurological complications. Patients with neurodegenerative or cerebrovascular disease are more likely to develop delirium [34]. Well-known risk factors of delirium are age, previous dementia history, disease severity during ICU stay, which had shown similar tendencies in our study (Table S2) [10]. Furthermore, patients with cerebrovascular diseases are at a higher risk of stroke recurrence during treatment in the ICU. In our study, two of three patients who had acute stroke during ICU stay had a history of cerebrovascular disease (data not shown in the tables).

The APACHE II and SOFA scores are useful tools in predicting the clinical outcomes of critically ill patients, and Zhou et al. showed that a higher SOFA score at admission was a risk factor for death in adult patients with COVID-19 [35,36]. Our findings showed that the APACHE II or SOFA score was an independent factor of ICU mortality in COVID-19 patients admitted to the ICU.

This study included all patients from the opening to the closing of the ICU in a government-designated hospital, and we were able to obtain the clinical outcomes of all patients, which are both the strengths of this study. Nevertheless, this study has several limitations. Careful attention should be paid to the interpretation of the results as this is a retrospective study with a small number of patients. Functional status prior to ICU admission such as pre-modified Rankin scale score, which could affect clinical outcome, was not reflected in the analysis because of the limitation of a retrospective study. In addition, we were unable to ascertain causality. New-onset neurological complications could be correlated with other medical complications and ICU death, directly or indirectly; however, these correlations could not confirm causation. Some of the neurological complications could have also been underdiagnosed, such as non-convulsive seizures and unexplained encephalopathy, which are relatively common in ICU patients with sepsis [37]. Our study did not implement an EEG for all ICU patients, only those who developed seizures clinically or had unexplained mental changes. In addition, only stable patients with vital signs underwent MRI. A recent study conducted brain MRIs on 13 patients with unexplained encephalopathic features and found two cases of acute and one case of subacute cerebral infarction; however, neither of the cases had any focal symptoms of a stroke [7].

\section{Conclusions}

In this study, we observed that more than one-third of the patients who were treated in the integrated ICU for COVID-19 infection already had neurological comorbidities. Furthermore, new-onset neurological complications occurred in $44.2 \%$ of the patients during ICU stay. Because preexisting neurological comorbidities and new-onset neurological complications during ICU stay have significant effects on the treatment and prognosis of ICU patients, both directly and indirectly, clinical awareness about neurological problems is thought to be important for the treatment of patients hospitalized in the ICU due to COVID-19. Therefore, additional attention to neurological conditions is pertinent to treating COVID-19. This can be accomplished by including neurointensivists in frontline treatment teams.

Supplementary Materials: The following are available online at https:/ /www.mdpi.com/article/10 $.3390 / \mathrm{jcm} 10112281 / \mathrm{s} 1$, Table S1: Independent predictors associated with ICU mortality in multiple logistic regression analysis using SOFA score, Table S2: Baseline characteristics of 52 intensive care unit patients infected with COVID-19 with respect to the presence of delirium.

Author Contributions: Conceptualization, J.P. and J.-H.H.; methodology, J.P. and J.-H.H.; software, J.-H.H.; validation, J.P. and J.-H.H.; formal analysis, J.P. and J.-H.H.; investigation, Y.-S.K., H.-A.K., D.-H.K., and J.H.; data curation, J.P., S.-H.J., H.P., and S.-I.S.; writing-original draft preparation, J.P. and J.-H.H.; writing - review and editing, J.P., J.-H.H. and H.A.C.; supervision, J.-H.H. All authors have read and agreed to the published version of the manuscript. 
Funding: This research received no external funding.

Institutional Review Board Statement: The study was conducted according to the guidelines of the Declaration of Helsinki, and approved by the Institutional Review Board of Keimyung University Dongsan Hospital (IRB o. 2020-05-048).

Informed Consent Statement: Patient consent was waived by the IRB given the retrospective design of our study with anonymized clinical data.

Data Availability Statement: The data presented in this study are available on request from the corresponding author.

Conflicts of Interest: The authors declare no conflict of interest.

\section{References}

1. Huang, C.; Wang, Y.; Li, X.; Ren, L.; Zhao, J.; Hu, Y.; Zhang, L.; Fan, G.; Xu, J.; Gu, X.; et al. Clinical Features of Patients Infected with 2019 Novel Coronavirus in Wuhan, China. Lancet 2020, 395, 497-506. [CrossRef]

2. Mueller, A.L.; McNamara, M.S.; Sinclair, D.A. Why Does COVID-19 Disproportionately Affect Older People? Aging 2020, 12, 9959-9981. [CrossRef] [PubMed]

3. Davies, N.G.; Klepac, P.; Liu, Y.; Prem, K.; Jit, M.; Pearson, C.A.B.; Quilty, B.J.; Kucharski, A.J.; Gibbs, H.; Clifford, S.; et al. Age-Dependent Effects in the Transmission and Control of COVID-19 Epidemics. Nat. Med. 2020, 26, 1205-1211. [CrossRef]

4. People with Certain Medical Conditions. 2021. Available online: https://www.cdc.gov/coronavirus/2019-ncov/need-extraprecautions/people-with-medical-conditions.html (accessed on 5 April 2021).

5. Mao, L.; Jin, H.; Wang, M.; Hu, Y.; Chen, S.; He, Q.; Chang, J.; Hong, C.; Zhou, Y.; Wang, D.; et al. Neurologic Manifestations of Hospitalized Patients With Coronavirus Disease 2019 in Wuhan, China. Jama Neurol. 2020, 77. [CrossRef]

6. Oxley, T.J.; Mocco, J.; Majidi, S.; Kellner, C.P.; Shoirah, H.; Singh, I.P.; Leacy, R.A.D.; Shigematsu, T.; Ladner, T.R.; Yaeger, K.A.; et al. Large-Vessel Stroke as a Presenting Feature of Covid-19 in the Young. N. Engl. J. Med. 2020, e60. [CrossRef] [PubMed]

7. Helms, J.; Kremer, S.; Merdji, H.; Clere-Jehl, R.; Schenck, M.; Kummerlen, C.; Collange, O.; Boulay, C.; Fafi-Kremer, S.; Ohana, M.; et al. Neurologic Features in Severe SARS-CoV-2 Infection. N. Engl. J. Med. 2020, 23, 2268-2270. [CrossRef]

8. Interim Guidelines for Collecting, Handling, and Testing Clinical Specimens from Persons for Coronavirus Disease 2019 (COVID19). 2020. Available online: https://www.cdc.gov/coronavirus/2019-nCoV/lab/guidelines-clinical-specimens.html (accessed on 5 April 2021).

9. Hong, K.H.; Lee, S.W.; Kim, T.S.; Huh, H.J.; Lee, J.; Kim, S.Y.; Park, J.-S.; Kim, G.J.; Sung, H.; Roh, K.H.; et al. Guidelines for Laboratory Diagnosis of Coronavirus Disease 2019 (COVID-19) in Korea. Ann. Lab. Med. 2020, 40, 351-360. [CrossRef] [PubMed]

10. Devlin, J.W.; Skrobik, Y.; Gélinas, C.; Needham, D.M.; Slooter, A.J.C.; Pandharipande, P.P.; Watson, P.L.; Weinhouse, G.L.; Nunnally, M.E.; Rochwerg, B.; et al. Clinical Practice Guidelines for the Prevention and Management of Pain, Agitation/Sedation, Delirium, Immobility, and Sleep Disruption in Adult Patients in the ICU. Crit. Care Med. 2018, 46, e825-e873. [CrossRef]

11. World Health Organization. COVID-19 Clinical Management: Living Guidance. Available online: https://www.who.int/ publications/i/item/WHO-2019-nCoV-clinical-2021-1 (accessed on 25 January 2021).

12. Khwaja, A. KDIGO Clinical Practice Guidelines for Acute Kidney Injury. Nephron Clin. Pract. 2012, 120, c179-c184. [CrossRef]

13. Singer, M.; Deutschman, C.S.; Seymour, C.W.; Shankar-Hari, M.; Annane, D.; Bauer, M.; Bellomo, R.; Bernard, G.R.; Chiche, J.-D.; Coopersmith, C.M.; et al. The Third International Consensus Definitions for Sepsis and Septic Shock (Sepsis-3). JAMA 2016, 315, 801-810. [CrossRef] [PubMed]

14. KNAUS, W.A.; DRAPER, E.A.; WAGNER, D.P.; ZIMMERMAN, J.E. APACHE II: A Severity of Disease Classification System. Crit. Care Med. 1985, 13, 818-829. [CrossRef]

15. García-Azorín, D.; Martínez-Pías, E.; Trigo, J.; Hernández-Pérez, I.; Valle-Peñacoba, G.; Talavera, B.; Simón-Campo, P.; de Lera, M.; Chavarría-Miranda, A.; López-Sanz, C.; et al. Neurological Comorbidity Is a Predictor of Death in Covid-19 Disease: A Cohort Study on 576 Patients. Front. Neurol. 2020, 11, 781. [CrossRef] [PubMed]

16. Zhang, L.; Sun, W.; Wang, Y.; Wang, X.; Liu, Y.; Zhao, S.; Long, D.; Chen, L.; Yu, L. Clinical Course and Mortality of Stroke Patients With Coronavirus Disease 2019 in Wuhan, China. Stroke 2020, 51, 2674-2682. [CrossRef]

17. Cook, D.J.; Kollef, M.H. Risk Factors for ICU-Acquired Pneumonia. JAMA 1998, 279, 1605. [CrossRef] [PubMed]

18. Torres, A.; Peetermans, W.E.; Viegi, G.; Blasi, F. Risk Factors for Community-Acquired Pneumonia in Adults in Europe: A Literature Review. Thorax 2013, 68, 1057. [CrossRef] [PubMed]

19. Varelas, P.N.; Conti, M.M.; Spanaki, M.V.; Potts, E.; Bradford, D.; Sunstrom, C.; Fedder, W.; Bey, L.H.; Jaradeh, S.; Gennarelli, T.A. The Impact of a Neurointensivist-Led Team on a Semiclosed Neurosciences Intensive Care Unit\&ast. Crit. Care Med. 2004, 32, 2191-2198. [CrossRef] [PubMed]

20. Moriguchi, T.; Harii, N.; Goto, J.; Harada, D.; Sugawara, H.; Takamino, J.; Ueno, M.; Sakata, H.; Kondo, K.; Myose, N.; et al. A First Case of Meningitis/Encephalitis Associated with SARS-Coronavirus-2. Int. J. Infect. Dis. 2020, 94, 55-58. [CrossRef]

21. Munz, M.; Wessendorf, S.; Koretsis, G.; Tewald, F.; Baegi, R.; Krämer, S.; Geissler, M.; Reinhard, M. Acute Transverse Myelitis after COVID-19 Pneumonia. J. Neurol. 2020, 267, 2196-2197. [CrossRef] [PubMed] 
22. Toscano, G.; Palmerini, F.; Ravaglia, S.; Ruiz, L.; Invernizzi, P.; Cuzzoni, M.G.; Franciotta, D.; Baldanti, F.; Daturi, R.; Postorino, P.; et al. Guillain-Barré Syndrome Associated with SARS-CoV-2. N. Engl. J. Med. 2020, 382, 2574-2576. [CrossRef]

23. Parsons, T.; Banks, S.; Bae, C.; Gelber, J.; Alahmadi, H.; Tichauer, M. COVID-19-Associated Acute Disseminated Encephalomyelitis (ADEM). J. Neurol. 2020, 1-4. [CrossRef] [PubMed]

24. Poyiadji, N.; Shahin, G.; Noujaim, D.; Stone, M.; Patel, S.; Griffith, B. COVID-19-Associated Acute Hemorrhagic Necrotizing Encephalopathy: CT and MRI Features. Radiology 2020, 296, 201187. [CrossRef]

25. Lu, L.; Xiong, W.; Liu, D.; Liu, J.; Yang, D.; Li, N.; Mu, J.; Guo, J.; Li, W.; Wang, G.; et al. New Onset Acute Symptomatic Seizure and Risk Factors in Coronavirus Disease 2019: A Retrospective Multicenter Study. Epilepsia 2020, 61, e49-e53. [CrossRef] [PubMed]

26. Ellul, M.A.; Benjamin, L.; Singh, B.; Lant, S.; Michael, B.D.; Easton, A.; Kneen, R.; Defres, S.; Sejvar, J.; Solomon, T. Neurological Associations of COVID-19. Lancet Neurol. 2020, 19, 767-783. [CrossRef]

27. Hess, D.C.; Eldahshan, W.; Rutkowski, E. COVID-19-Related Stroke. Transl. Stroke Res. 2020, 11, 322-325. [CrossRef]

28. Tang, N.; Li, D.; Wang, X.; Sun, Z. Abnormal Coagulation Parameters Are Associated with Poor Prognosis in Patients with Novel Coronavirus Pneumonia. J. Thromb. Haemost. 2020, 18, 844-847. [CrossRef]

29. Kwon, D.H.; Do, Y.; Eun, M.-Y.; Lee, J.; Park, H.; Sohn, S.-I.; Hong, J.-H. Characteristics of Acute Stroke in Patients with Coronavirus Disease 2019 and Challenges in Stroke Management during an Epidemic. J. Korean Med. Sci. 2020, 35 , e324. [CrossRef]

30. Murthy, S.; Gomersall, C.D.; Fowler, R.A. Care for Critically Ill Patients With COVID-19. Jama 2020, 323, 1499-1500. [CrossRef]

31. Long, B.; Brady, W.J.; Koyfman, A.; Gottlieb, M. Cardiovascular Complications in COVID-19. Am. J. Emerg. Med. 2020. [CrossRef] [PubMed]

32. Ouimet, S.; Kavanagh, B.P.; Gottfried, S.B.; Skrobik, Y. Incidence, Risk Factors and Consequences of ICU Delirium. Intens. Care Med. 2007, 33, 66-73. [CrossRef] [PubMed]

33. Ely, E.W.; Shintani, A.; Truman, B.; Speroff, T.; Gordon, S.M.; Harrell, J.F.E.; Inouye, S.K.; Bernard, G.R.; Dittus, R.S. Delirium as a Predictor of Mortality in Mechanically Ventilated Patients in the Intensive Care Unit. Jama 2004, 291, 1753-1762. [CrossRef] [PubMed]

34. Inouye, S.K.; Westendorp, R.G.; Saczynski, J.S. Delirium in Elderly People. Lancet 2014, 383, 911-922. [CrossRef]

35. Zhou, F.; Yu, T.; Du, R.; Fan, G.; Liu, Y.; Liu, Z.; Xiang, J.; Wang, Y.; Song, B.; Gu, X.; et al. Clinical Course and Risk Factors for Mortality of Adult Inpatients with COVID-19 in Wuhan, China: A Retrospective Cohort Study. Lancet 2020, 395, $1054-1062$. [CrossRef]

36. Vincent, J.-L.; de Mendonca, A.; Cantraine, F.; Moreno, R.; Takala, J.; Suter, P.M.; Sprung, C.L.; Colardyn, F.; Blecher, S. Use of the SOFA Score to Assess the Incidence of Organ Dysfunction/Failure in Intensive Care Units. Crit. Care Med. 1998, 26, 1793-1800. [CrossRef] [PubMed]

37. Oddo, M.; Carrera, E.; Claassen, J.; Mayer, S.A.; Hirsch, L.J. Continuous Electroencephalography in the Medical Intensive Care Unit. Crit. Care Med. 2009, 37, 2051-2056. [CrossRef] [PubMed] 